\title{
Proteomics Approach To Trace Site-Specific Damage in Aquatic Extracellular Enzymes During Photoinactivation
}

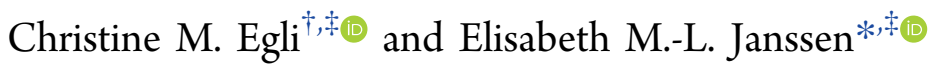 \\ ${ }^{\dagger}$ Institute of Biogeochemistry and Pollutant Dynamics, ETH Zurich, Zürich, 8092, Switzerland \\ ${ }^{\ddagger}$ Swiss Federal Institute of Aquatic Science and Technology, Eawag, Dübendorf, 8600, Switzerland
}

Supporting Information

\begin{abstract}
Extracellular enzymes are major drivers of biogeochemical nutrient and carbon cycling in surface water. While photoinactivation is regarded as a major inactivation process of these enzymes, the underlying molecular changes have received little attention. This study demonstrates how light exposure leads to a rapid loss of phosphatase, aminopeptidase, and glucosidase activities of biofilm samples and model enzymes. Here, an optimized proteomics approach allowed simultaneous observation of inactivation and molecular changes. Site-specific fingerprints of degradation kinetics have been generated and visualized in the three-dimensional proteins. Oxidation of tryptophan, the chromophoric target, initiated

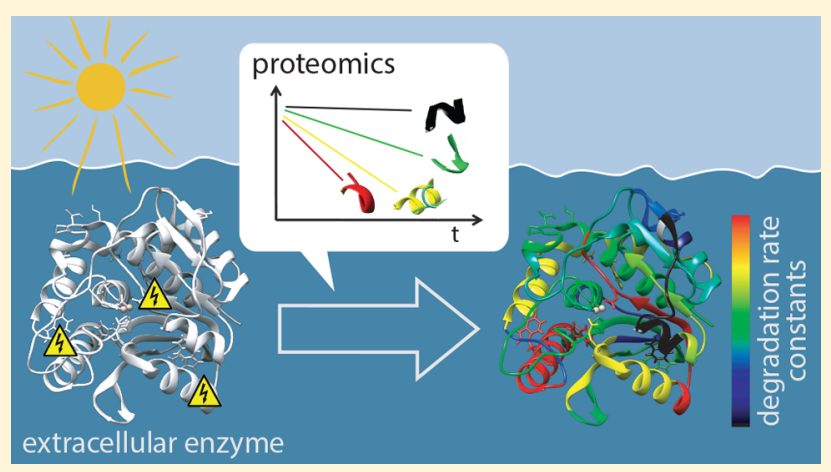
secondary reactions. Evidence was obtained that tyrosine residues act as intramolecular antioxidants, reflected in decelerated decay of tryptophan-containing peptides and enhanced decay of tyrosine-containing peptides. In addition, subsequent methionine oxidation and disulfide reduction contribute to heterogeneous photodamage. The proximity to tryptophan residues explains $>95 \%$ of the photodamage across the protein structures. The presence of redox active organic matter or a model antioxidant in solution quenched not only photoinactivation and tryptophan oxidation but also all subsequent damage. The developed analytical approach can be applied to other research questions in environmental sciences where site-specific damage in a protein is essential.
\end{abstract}

\section{INTRODUCTION}

Extracellular enzymes are important drivers of biogeochemical cycles in various environmental compartments as they often initiate the remineralization of macromolecular detritus. ${ }^{1}$ The enzymes are released by heterotrophic microorganisms to the environment via active secretion or cell lysis. ${ }^{2}$ Once released, extracellular enzymes are exposed to numerous environmental factors that can lead to their inactivation. The activity of an enzyme depends critically on the integrity of its macromolecular structure. Inactivation of extracellular enzymes and therefore changes in their structure need to be better understood to predict their impacts on biogeochemical cycles. ${ }^{3}$ Regarding the inactivation in surface waters, exposure to sunlight presents one major decay pathway for proteins harboring photolabile amino acids.

Phosphatase, aminopeptidase, and glucosidase are ubiquitous classes of extracellular enzymes, and their activities can be commonly detected in surface waters. ${ }^{3,4}$ Previous studies have shown that these enzymes are photochemically inactivated by sunlight. $^{5-7}$ The photoinactivation of the model enzyme Escherichia coli phosphatase was found to be slowed down in the presence of chromophoric dissolved organic matter (CDOM). ${ }^{7,8}$ The indole moiety of tryptophan (Trp) presents the dominant chromophore within proteins. Upon absorption of UVB light, tryptophan photoionizes to a tryptophan radical cation, which leads to degradation of tryptophan., ${ }^{9}$ Recent work suggests a link between photooxidative damage of tryptophan residues and the apparent decelerating effect on photoinactivation by organic matter. ${ }^{11}$ In the presence of redox active DOM or natural antioxidants, photodegradation of freely dissolved tryptophan can be slowed down by reducing the radical intermediate back to tryptophan. ${ }^{12}$

While Trp-induced photooxidation is generally believed to be a major decay pathway of proteins in surface waters, previous studies focused on monitoring activities rather than structural changes in the molecules. Generally, photooxidation reactions have been studied in short peptides or free amino acids, e.g., regarding the interaction of photochemically excited tryptophan and tyrosine. ${ }^{13-15}$ Studies that investigated subsequent reactions in proteins observed intramolecular reactions including tyrosine oxidation by tryptophan radical intermediates and disulfide reduction by excited triplet state tryptophan (see Figure 1A-E). ${ }^{16-20}$ These studies demonstrated that subsequent reactions are critically dependent on the mircoenvironment of the tryptophan residues, where the

Received: December 14, 2017

Revised: June 6, 2018

Accepted: June 19, 2018

Published: June 19, 2018 


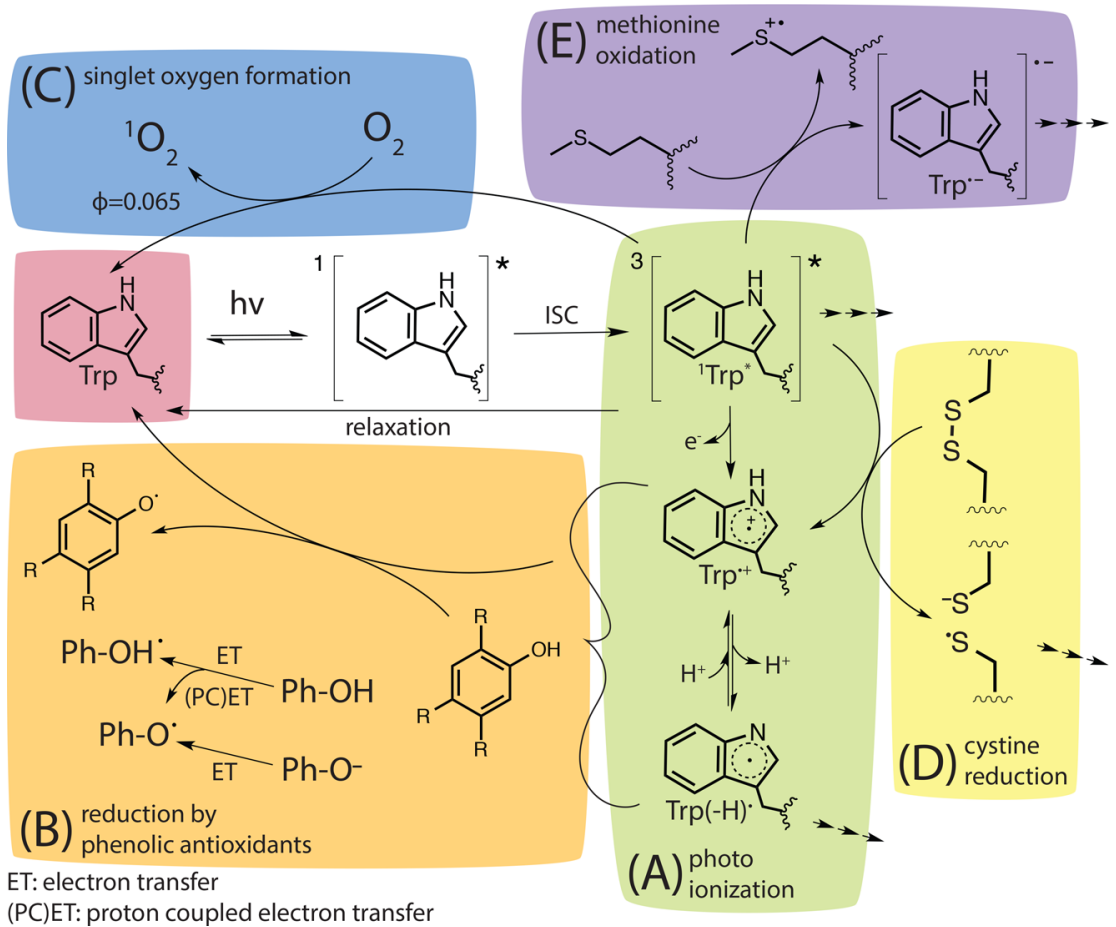

Figure 1. Trp-mediated intermolecular reaction pathways in amino acid-based molecules. Tryptophan absorbs light in the UVB range and forms a triplet via intersystem crossing (ISC) which can subsequently lead to radical formation by photoionization (A). These radicals can be reduced back to tryptophan via (PC)ET by suitable phenolic antioxidants (B). Triplet tryptophan can subsequently form singlet oxygen recovering ground state tryptophan (C), reduce neighboring disulfide bridges forming tryptophan radical intermediates (D), or oxidize methionine residues under radical anion formation (E). A more detailed description of the investigated reaction pathways can be found in Section S1.

photochemical reaction is initiated. Photodegradation is expected to start at the chromophore tryptophan, but its oxidation may not directly cause enzyme inactivation. Most likely, molecular damage accumulates during light exposure, and subsequent reactions initiated by tryptophan photochemistry eventually impair the function of the enzyme. Compared to studies with linear peptides, the analysis of Trpmediated photochemical damage in whole proteins comes with some additional challenges as high resolution of the protein structure and advanced methods to follow site-specific decay are crucial.

Proteins are often studied with traditional peptide-centric proteomics approaches, which aim to monitor targeted proteins or discover a whole proteome using a generic digestion protocol to detect a small number of characteristic and analytically promising peptides per protein. The assessment of site-specific structural changes in one specific protein requires resolution of the entire macromolecule, meaning that the full sequence has to be quantitatively detectable. Such resolution requires extensive optimization of the digestion protocol, chromatographic separation, and data analysis. While biochemical and biomedical applications frequently make use of high structural resolution proteomics techniques, such techniques have rarely been applied to follow degradation of enzymes in environmental systems. One recent example presents a study demonstrating the dependence of solvent accessibility of individual residues toward oxidation by photochemically produced singlet oxygen in a model protein. ${ }^{21}$

Here, we present a bottom-up proteomics approach to study intramolecular damage of enzymes and exemplify this by inactivation and molecular changes induced during light exposure. First, we confirm the detection and photochemical inactivation of phosphatase, aminopeptidase, and $\alpha$ - and $\beta$ glucosidase activities in natural biofilm samples. We demonstrate analogous inactivation behavior for the model enzyme Escherichia coli alkaline phosphatase (ECAP), Aeromonas proteolytica aminopeptidase (BLAP), and Bacillus stearothermophilus $\alpha$-glucosidase (GLU). We then performed a structural analysis for these model enzymes to resolve spatial and temporal damage in the protein structure. After exposure to light, we use a proteomics approach to (1) take the macromolecule apart into its peptide fragments, (2) determine the degradation rate constant of each peptide individually, and (3) assign site-specific fingerprints of degradation kinetics. As a result, we create protein maps that visualize the site-specific reaction rate constants in the macromolecule, pinpoint initial targets, and demonstrate the occurrence of secondary intramolecular reactions, which explain the majority of heterogeneous damage across the protein structures.

\section{EXPERIMENTAL SECTION}

Materials. All proteins and chemicals were purchased from commercial vendors. More details can be found in Section S2.

Colonization of Natural Biofilm. Periphyton biofilms were grown on glass slides in indoor flow-through channels continuously supplied with freshwater from a small stream in Dübendorf, Switzerland. ${ }^{22,23}$ Channels were operated at a constant flow rate of $0.05 \mathrm{~ms}^{-1}$ under a light/dark cycle of $12 \mathrm{~h}$ with BioSun fluorescent tubes (MLT Moderne Licht-Technik AG, Wettingen, Switzerland). The colonized glass slides were removed after 3 weeks, and the biofilm was harvested by removing the deposit from the slides into a suspension of streamwater. The suspension was sonicated for $10 \mathrm{~min}$, centrifuged for $2 \mathrm{~min}$ (16000 rpm/rcf), and filtered (PFFE, 
$0.22 \mu \mathrm{m}$ ) with a final $\mathrm{pH}$ around 8 . Biofilm suspension did not show any interfering signals with the absorbance/fluorescence of the enzyme activity assays.

Photochemical Experiments. Light exposure was carried out in a photoreactor (Rayonet Southern New England Ultraviolet Co.) with UVB light (RPR-3000 A bulbs) on a merry-go-around turn table and by back-to-front exposure in quartz sample tubes for biofilm suspension and model enzyme samples, respectively. The light intensity was adjusted relative to the inactivation of the individual samples to capture enough decay for regression analysis. Therefore, biofilm suspension, phosphatase, and aminopeptidase enzyme solutions were exposed to the light of 8 UVB bulbs, and glucosidase enzyme solutions were exposed using only 2 UVB bulbs because of significantly faster decay. Light fluxes were quantified by actinometry $(10 \mu \mathrm{M} p$-nitroanisole and $0.5 \mathrm{mM}$ pyridine, details in Section S3) with 6 UVB bulbs, which equates to 9.5fold of the UVB intensity (between 270 and $320 \mathrm{~nm}$ ) of the solar spectrum. Activity data was normalized to the same light intensity for comparison (see Figure 2).

Direct Inactivation of Natural and Model Enzymes. Biofilm suspensions were used after filtration as described above. Time series samples were subjected to phosphatase, aminopeptidase, and $\alpha$ - and $\beta$-glucosidase activity assays as described below. Activities of model enzymes were measured in the presence and absence of $50 \mu \mathrm{M}$ esculetin and $10 \mathrm{mg}_{\mathrm{C}} \mathrm{L}^{-1}$ NOM. The NOM types were Waskish Peat, Aldrich Humic acid, and Nordic Lake for ECAP, BLAP, and GLU, respectively. The observed inactivation rate constants of model enzymes in the presence of esculetin and NOM were corrected for light screening effects as described in detail elsewhere. ${ }^{11}$ The model enzymes were additionally exposed to simulated sunlight for comparison of inactivation rate constants (Figure S11.1).

Indirect Inactivation with Singlet Oxygen. Indirect photochemical inactivation in the presence of ${ }^{1} \mathrm{O}_{2}$ was conducted for BLAP and GLU as previously described for ECAP. ${ }^{11}$ Briefly, solutions containing the model enzyme, furfuryl alcohol (40 $\mu \mathrm{M})$, and Rose Bengal ( $3 \mu \mathrm{M}$ ) were exposed to visible light (Xe lamp with $455 \mathrm{~nm}$ cutoff filter). Time series samples were analyzed for enzyme activity as described below. The ${ }^{1} \mathrm{O}_{2}$ steady state concentration was quantified using furfuryl alcohol. $^{24}$ The bimolecular inactivation rate constant was calculated as the ratio of observed inactivation rate constant and ${ }^{1} \mathrm{O}_{2}$ concentration (details in Section S4).

Direct Site-Specific Degradation. In proteomics experiments, enzyme solutions with ECAP, BLAP, and GLU were exposed to UVB light during 60, 100, and $45 \mathrm{~min}$, respectively, and for each experiment, a time series of at least 6 samples was taken and subjected to proteomics and activity measurements as described below. The enzyme solutions contained $>0.1$ $\mu \mathrm{g} \mu \mathrm{L}^{-1}$ in $50 \mathrm{mM}$ ambic at $\mathrm{pH} 8$ with and without the addition of the antioxidant esculetin $(100 \mu \mathrm{M})$.

UPLC-Based Enzyme Activity Assays. The activities of phosphatase, aminopeptidase, and $\alpha$ - and $\beta$-glucosidase were determined for biofilm and model enzyme solutions using umbelliferone- and nitrophenyl-based substrates. ${ }^{11}$ Preliminary tests were carried out to determine the substrate concentrations for maximum rates, i.e., when the reaction is not limited by substrate availability. After defined incubation times of enzyme sample with substrate (10-60 min), enzyme activities were stopped by acidifying the samples, ensuring that substrate and product stability was maintained. The respective hydrolysis products were separated by liquid chromatography with a C18 column and quantified with fluorescence or absorbance detection. Inactivation rate constants were determined from linear regression of the log-normalized activities over time. Where applicable, an initial lag-phase of inactivation was considered with a shoulder log-linear decay model. $^{25}$ A more detailed description of the MichaelisMenten kinetics, activity assays, and the shoulder log-linear decay model can be found in Sections S5-S7.

Proteomics Workflows. Sample preparation was optimized for each enzyme and consisted of steps for (1) metal chelation, (2) reduction of the disulfide bridges, (3) alkylation of thiols, and (4) digestion. ${ }^{21,26,27}$ Samples contained enzyme concentrations between 0.117 and $0.130 \mu \mathrm{g} \mu \mathrm{L}^{-1}$. The sample preparation workflow for ECAP consisted of (1) metal chelation with EDTA, (2) reduction of the disulfide bridges with DTT (sonication at $\left.60{ }^{\circ} \mathrm{C}\right)$, (3) alkylation of thiols with iodacetamide (at $37^{\circ} \mathrm{C}$ ), and (4) microwave-assisted digestion with trypsin. If not mentioned differently, chemicals and conditions were the same for all other enzymes. Compared to ECAP, BLAP was exposed to higher temperatures. After step (1), BLAP samples were heated up to $95^{\circ} \mathrm{C}$ and step (2) was conducted at $85{ }^{\circ} \mathrm{C}$ in the sonicator. Additionally, a surfactant (ProteaseMAX) was added in step (3), and chymotrypsin was used for an overnight digestion in step (4). In the sample preparation workflow for GLU, step (1) was omitted; step (2) was also conducted at $85{ }^{\circ} \mathrm{C}$, and an additional step with the surfactant was performed (also at $85{ }^{\circ} \mathrm{C}$ ). Step (3) for GLU was the same as for ECAP, and for the overnight digestion, trypsin was used (4). More details can be found in Section S8.

Peptide Analysis with Liquid Chromatography High Resolution Mass Spectrometry (LC-HRMS). Digested phosphatase samples $(5 \mu \mathrm{L})$ were injected to a nUPLC (nanoAcquity, Waters) equipped with a $5 \mu \mathrm{L}$ sample loop, a C18 trap column (Waters Nano Acquity UPLC, 2G-VMTrap, $5 \mu \mathrm{m}, 180 \mu \mathrm{m} \times 20 \mathrm{~mm}$ ), and a C18 column (Nano Acquity UPLC, BEH130, $1.7 \mu \mathrm{m}, 300 \mu \mathrm{m} \times 150 \mathrm{~mm}$, Waters) at a flow rate of $5 \mu \mathrm{L} \mathrm{min}{ }^{-1} \cdot{ }^{21,26}$ Digested aminopeptidase and glucosidase samples $(5 \mu \mathrm{L})$ were injected to a $\mu \mathrm{UPLC}$ (Ultimate 3000, Dionex) with the same setting. Peptide separation was obtained with a gradient of A (95\% MQ 5\% acetonitrile, $0.1 \%$ formic acid) and B (95\% acetonitrile, 5\% $\mathrm{MQ}, 0.1 \%$ formic acid). After chromatographic separation, peptides were detected with a high resolution Orbitrap mass spectrometer (nanoAcquity coupled to Thermo Exactive or Ultimate 3000 to a Thermo Q Exactive Plus) equipped with an electron spray ionization source (see Section S9 for detailed settings and gradients).

Data Analysis. Peptides identification and quantification were performed with Skyline (v3.5 and v3.6). ${ }^{28}$ For the identification of targeted peptides, the in silico predicted isotope distribution and the presence of multiple charge states were used. Figures in Section S16 show the progression of the chromatographic traces of the most intense charge state, the total mass spectra, and an inset for one or two charge states for all studied peptides. To quantify peptide degradation, peak areas $(A)$ were normalized to the respective initial areas, $\ln (A$ / $\left.A_{0}\right)$, plotted log-normalized versus exposure time, and fitted to a linear regression model where the slope represents the pseudo-first-order reaction rate constant. In the following, this experimentally obtained rate constant is referred to as the (observed) reaction rate constant or degradation rate constant. Whether the degradation of a peptide was detectable with our 

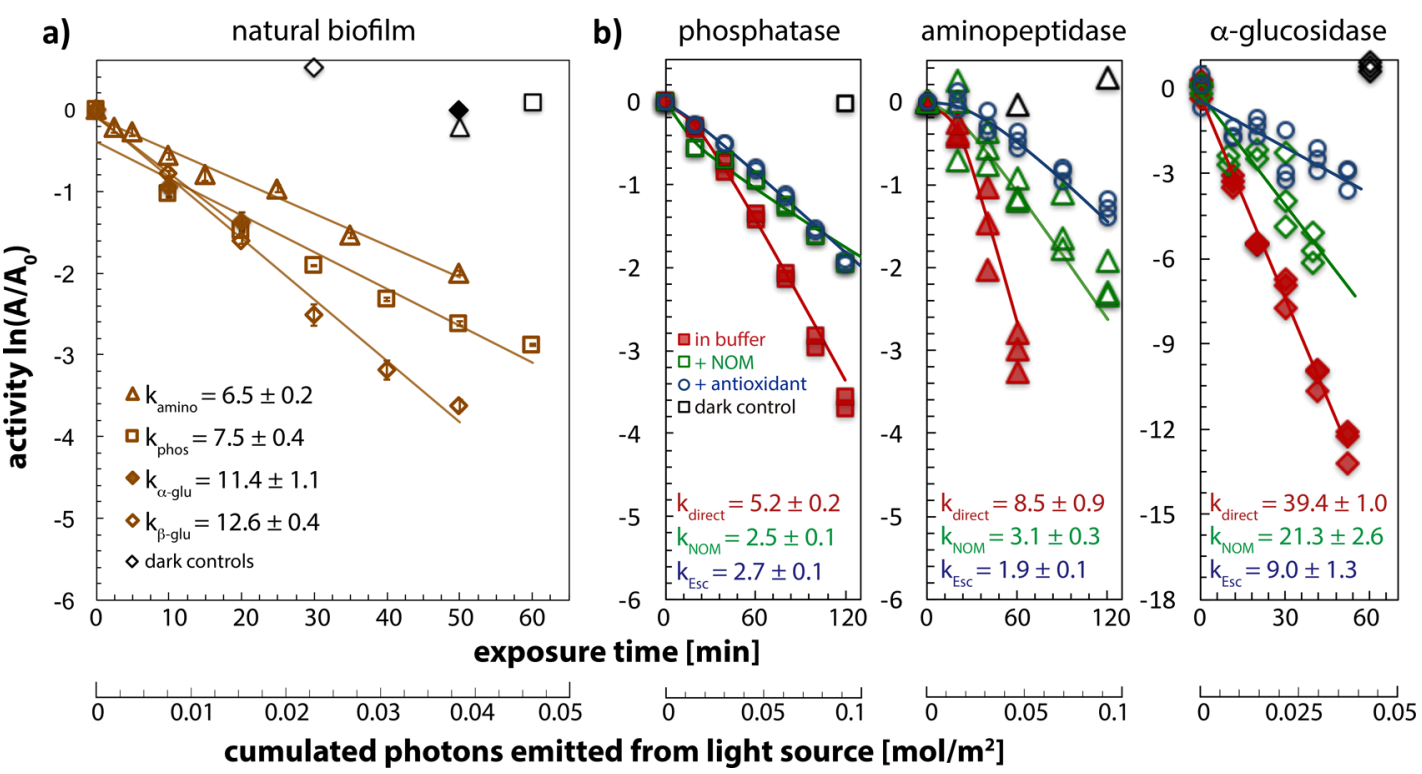

Figure 2. (a) Loss of activities in biofilm samples for phosphatases (squares), aminopeptidases (triangles), and $\alpha$ - and $\beta$-glucosidases (empty and filled diamonds, respectively) during UVB light exposure. (b) Loss of model enzyme activities for extracellular phosphatase (ECAP), aminopeptidase (BLAP), and $\alpha$-glucosidase (GLU) during exposure to UVB light in buffered solution (red, filled symbols), in the presence of natural organic matter (NOM, $10 \mathrm{mg}_{\mathrm{C}} \mathrm{L}^{-1}$, green, open symbols), and model antioxidant esculetin (Esc, $50 \mu \mathrm{M}$, blue, open circles). The NOM types were Waskish Peat, Aldrich Humic acid, and Nordic Lake for ECAP, BLAP, and GLU, respectively. All NOM types showed comparable effects on inactivation for the model enzymes (data not shown). Inactivation data was light screening corrected. The respective inactivation rate constants, $k \pm$ standard deviation $\times 10^{-4} \mathrm{~s}^{-1}$, were obtained by (shoulder) log-linear regression. Controls are indicated with black symbols. The cumulated photons emitted between 270 and $320 \mathrm{~nm}$ are indicated on the secondary $x$-axis in accordance to respective exposure time. Data were normalized to the light intensity of 6 UVB bulbs (unnormalized data is presented in Figure S11.2).

method was evaluated on the basis of the goodness of the linear regression $\left(R^{2}\right)$ and the accuracy of the estimate of the slope. Data that showed regression with $R^{2}<0.6$ and slopes with standard error larger than $40 \%$ were omitted; their degradation rate constant was labeled as not detected, n.d. (see Tables S10.1-S10.3), and colored in black (Figures 3 and S10.1-S10.5). Among all peptides, $>75 \%$ showed an $R^{2}>0.8$ and a standard deviation of the degradation rate constant $<20 \%$.

To study site-specific relations within the macromolecular structures of the enzymes, the reaction rate constants were visualized in three-dimensional representations using the software UCSF Chimera. ${ }^{29}$ Chimera was further used to determine distances between reactive residues in the previously published crystal structures of the studied proteins. ${ }^{30-32}$

\section{RESULTS AND DISCUSSION}

Photoinactivation of Enzymes from Natural Biofilm. We detected phosphatase, aminopeptidase, and $\alpha$ - and $\beta$ glucosidase activities in filtered freshwater biofilm samples harvested from stream channels. Data in Figure 2a show that UVB light exposure led to a rapid loss of activity for all four measured enzyme classes with apparent first-order kinetics. The glucosidase activity was significantly more sensitive to light exposure with approximately 1 order of magnitude faster decay kinetics. Our observations are consistent with previous findings of photoinactivation of phosphatase from natural biofilm samples as well as phosphatase, aminopeptidase, and $\beta$ glucosidase in natural seawater samples and model enzymes. ${ }^{5,11}$ Such natural surface water contains chromophoric dissolved organic matter (CDOM), which can contribute to enzyme inactivation by indirect photochemical processes involving various photochemically produced reactive inter- mediates. The measured inactivation is therefore a result of the combination of direct and indirect photochemical processes. Another layer of complexity is added when considering the dual roles of redox active DOM, which can also function as natural antioxidant during direct photoinactivation. ${ }^{11,12}$ Here, we were particularly interested in disentangling some of these co-occurring reactions. Therefore, we continued our studies with structurally resolved model enzymes to investigate the site-specific molecular damage in the protein structure by direct light exposure. We then added DOM or model antioxidants to investigate their effects on inactivation and molecular damage.

Photochemical Inactivation and Degradation of Model Enzymes and the Effect of Antioxidants. We compared inactivation of enzymes from heterogeneous biofilm samples with single model enzymes in buffered nanopure water with and without the addition of CDOM $\left(10 \mathrm{mg}_{\mathrm{C}} \mathrm{L}^{-1}\right)$. Our data demonstrate that all model enzymes were rapidly inactivated by exposure to enhanced UVB light with glucosidase being inactivated 2 -fold faster, similar to observations of glucosidases in the biofilm samples (see Figure $2 \mathrm{a}, \mathrm{b})$. Similar results were obtained during exposure to simulated sunlight (see Figure S11.1). In the presence of CDOM or a model antioxidant, the inactivation was decelerated significantly, still after accounting for light screening effects. In the presence of $10 \mathrm{mg}_{\mathrm{C}} \mathrm{L}^{-1}$ CDOM, the inactivation rate constants were reduced by up to $63 \%$ and the model antioxidant esculetin $(50 \mu \mathrm{M})$ had a similar effect with deceleration by $48-78 \%$ (Table S11.1). These results demonstrate that substantial quenching effects can be observed across these enzymes. Hence, it can be generally expected that the presence of natural CDOM and antioxidants affects the photoinactivation of enzymes in sunlit surface waters. Previous 


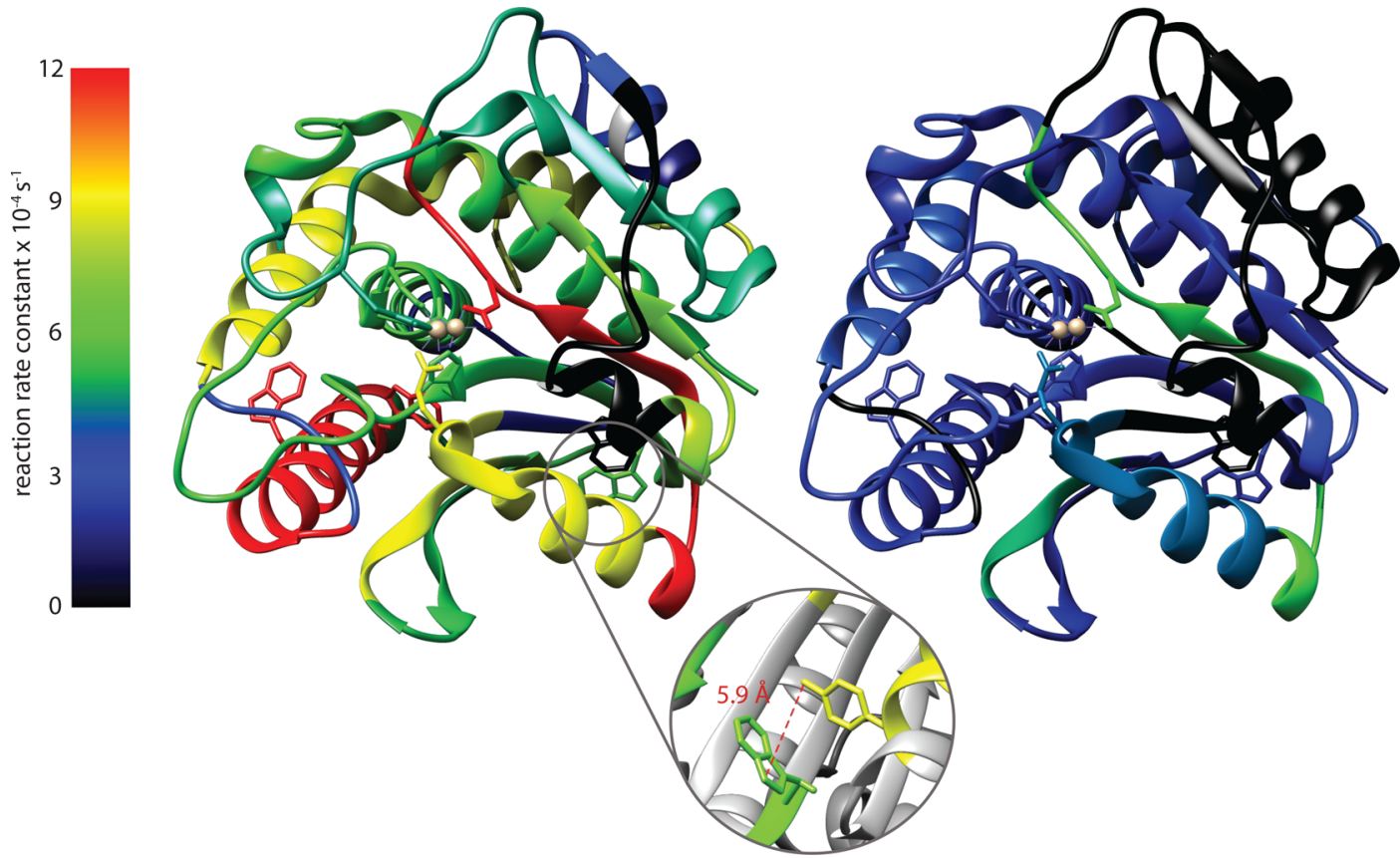

Figure 3. Color maps of reaction rate constants for Aeromonas proteolytica aminopeptidase (BLAP, EC 3.4.11.10, PDB 1RTQ) obtained during exposure to UVB light in the absence (left) and presence (right) of an antioxidant (esculetin). The inset exemplifies the determination of interresidue distance between the amine nitrogen of Trp91 and the phenol oxygen of Tyr165. Tabulated data for this color map can be found in Table S10.1. Fingerprints for phosphatase (ECAP) and $\alpha$-glucosidase (GLU) are presented in Figures S10.2 and S10.4. Color maps of all enzymes in an alternative color scale can also be found in Section S10.

irradiation experiments with ECAP showed that not only the inactivation decelerates but also the Trp-like fluorescence of the enzyme solutions was more stable in the presence of antioxidants, indicating more stable tryptophan residues. ${ }^{11} \mathrm{~A}$ similar response of Trp-like fluorescence was observed for BLAP and GLU (data not shown). To verify these assumptions across enzymes, we followed site-specific molecular damage of the enzyme structures by proteomics techniques in addition to inactivation. Furthermore, investigation of site-specific decay may resolve underlying mechanisms for heterogeneous damage of the protein structures initiated by tryptophan photooxidation.

Intramolecular Damage of Model Enzymes. To comprehensively evaluate the site-specific molecular damage, we applied a proteomics approach to the well-defined system of model enzymes and model antioxidant. Irradiation of the enzyme solutions with enhanced UVB light led to a heterogeneous distribution of molecular damage within the enzyme structures. Figure 3 shows the photochemical degradation for the aminopeptidase illustrated as a color map of site-specific reaction rate constants. Warmer colors, from yellow to red, indicate faster degradation rate constants of a peptide fragment compared to cooler colors, from green to blue, indicating slower decay. While some regions within the aminopeptidase did not degrade during light exposure (black areas), others had half-lives on the order of a few minutes. With our refined proteomics approach, we obtained full coverage of the peptide sequences for aminopeptidase (BLAP) and phosphatase (ECAP) and an $80 \%$ coverage for $\alpha$ glucosidase (GLU). Color maps and reaction rate constants for ECAP and GLU can be found in Figures S10.2 and S10.4 and Tables S10.2 and S10.3).

Compared to the inactivation rate constants, individual peptide decay was in the same order of magnitude. We analyzed the peptide degradation rate constants normalized to the inactivation rate constants, $k_{\text {peptide }} / k_{\text {activity }}$ (see Figure S12.1). On average, $28 \%$ of the peptides decayed faster than inactivation occurred $\left(k_{\text {peptide }} / k_{\text {activity }}>1\right)$ and $34 \%$ degraded significantly slower $\left(k_{\text {peptide }} / k_{\text {activity }}<0.5\right)$. As expected, damage of one peptide does not directly lead to the inactivation of the enzymes, and it is more likely that accumulation of damage eventually leads to loss of overall functionality.

In the presence of the antioxidant esculetin in bulk solution, we observed only minor photochemical degradation illustrated by a "cooling off" in the color map. The representation in Figure 3 on the right shows a shift toward cooler colors with a larger fraction of blue, which represents the lowest degradation rate constants of the peptides. We observed this "cooling off" effect and its correlation with retarded inactivation also for the model enzymes phosphatase and glucosidase (Figures S10.2 and S10.4). The generally observed decelerating effects of antioxidants on peptide degradation and inactivation support the hypothesis that similar photochemical reaction mechanisms occur across the macromolecular structures of different proteins. Similar decelerating effects were reported for freely dissolved monomeric tryptophan in the presence of increasing DOM or esculetin concentrations. ${ }^{12}$ Our observations suggest that the reaction mechanisms of monomeric tryptophan are comparable to tryptophan residues in the protein structure. Consequently, we have strong evidence that tryptophan radical intermediates formed from proteinogenic tryptophan can also be reduced by an external suitable electron donor like esculetin (pathways A and B in Figure 1). A dependency of the tryptophan residue solvent accessibility and the ability to quench its oxidation by esculetin might be expected as the antioxidant is supplied to the bulk solution as previously reported for histidine oxidation in proteins by ${ }^{1} \mathrm{O}_{2}$ in bulk solution. ${ }^{21}$ In contrast, no dependence on solvent accessibility 
of tryptophan residues for the participation in quenching reactions by antioxidants was observed here (see Figure S13.1).

Tryptophan Is the Initial Target of Protein Photooxidation. Tryptophan is the major chromophore in amino acid-based molecules and therefore expected to be the initial target of direct photooxidation. Photochemically formed radical intermediates, $\operatorname{Trp}^{\bullet+}$ and $\operatorname{Trp}(-\mathrm{H})^{\bullet}$, lead to further radical-mediated reactions and degradation of the Trpcontaining peptides (see Figure 1A). We found a decrease in Trp-like fluorescence of the bulk solution during exposure to light following pseudo-first-order kinetics, indicating that tryptophan residues are being altered (Figure S14.1).

Effectively, in each model enzyme, we found the overall fastest degradation rate constants for a peptide that contains a tryptophan residue. Degradation rate constants for the fastest peptides were similar across proteins, $(2.76 \pm 0.19) \times 10^{-3} \mathrm{~s}^{-1}$ for phosphatase (ECAP), (1.14 \pm 0.4$) \times 10^{-3} \mathrm{~s}^{-1}$ for aminopeptidase (BLAP), and $(1.46 \pm 0.28) \times 10^{-3} \mathrm{~s}^{-1}$ for glucosidase (GLU), after accounting for differences in light intensities during the experiments. The remaining differences in degradation rate constants are likely related to properties influenced by the microenvironment of the residue, e.g., variation in photochemical quantum yields and shifts in the tryptophan absorbance.

In the case of phosphatase, all peptides that contained one of the three tryptophan residues were by far the fastest degrading peptides within the protein structure. In aminopeptidase and glucosidase, not all Trp-containing peptides showed fast degradation rate constants and damage went beyond tryptophan oxidation for all enzymes (Figures 3, S10.2, and S10.4). Here, the local microenvironment of the tryptophan residues was inspected. Specifically, we investigate the hypotheses (i) that tyrosine acted as an intramolecular antioxidant and that triplet state tryptophan mediated (ii) disulfide reduction, (iii) methionine oxidation, and (iv) intramolecular ${ }^{1} \mathrm{O}_{2}$ formation causing further damage. We find that $>95 \%$ of the molecular damage can be attributed to the microenvironment of reactive tryptophan residues.

Tyrosine Acted as an Intramolecular Antioxidant: (PC)ET between $\operatorname{Trp}^{\bullet+}$ and Tyr. In proteins, intramolecular tyrosine residues (Tyr) can reduce neighboring photoionized tryptophan, similar to model antioxidants in bulk solution. Here, tyrosine can transfer electrons to $\operatorname{Trp}^{\bullet+}$ via a direct or a PCET to form ground state tryptophan (Figure 1B). Such redox reaction between $\operatorname{Trp}^{\bullet+}$ and tyrosine would result in a decelerated degradation of the Trp-containing peptides and an enhanced degradation of the affected Tyr-containing peptides. For this redox reaction to occur, the distance between the electron accepting Trp- $\mathrm{N}$ and the donating Tyr$\mathrm{O}, d_{\mathrm{Tr}-\mathrm{N}, \mathrm{Tyr}-\mathrm{O}}$, was previously suggested to be within 5.6 $\AA^{20,33,34}$ Therefore, we determined the inter-residue distance between the Trp-N and the Tyr-O in the crystal structures of the proteins, $d_{\text {Trp-N, Tyr-O }}$, and inspected the relationship to the observed reaction rate constants, $k_{\mathrm{obs}}$, for each pair of tryptophan and its nearest tyrosine. For comparison, we included relative reaction rate constants, $k_{\text {rel }}$ that represent the $k_{\text {obs }}$ value of each peptide, normalized by $k_{\text {obs }}$ of the fastest decaying peptide of the respective enzyme. An intramolecular antioxidant effect can be observed when $k_{\text {rel }}$ of the Trpcontaining peptide, $k_{\text {rel,Trp }}$, is decreased. Data in Table 1 show indeed reduced $k_{\text {rel, Trp }}$ values below 0.6 only for those tryptophan residues that are in close proximity to a
Table 1. The Position in the Primary Amino Acid Sequence, \#Trp, the Observed Reaction Rate Constant, $k_{\text {obs, Trp }}$, and Reaction Rate Constant Relative to the Fastest Decaying Peptide, $k_{\text {rel, Trp }}$, for Each Tryptophan Residue ${ }^{a}$

$\begin{array}{cccccc}\text { enzyme } & \text { \#Trp } & k_{\text {obs, Trp }}\left[\times 10^{-4} \mathrm{~s}^{-1}\right] & k_{\text {rel, Trp }} & \# \text { Tyr } & d_{\text {Trp-N,Tyr-O }}[\AA] \\ \text { ECAP } & 268 & 27.57 \pm 1.93 & 1.00 & 64 & 7.46 \\ & 109 & 18.65 \pm 1.11 & 0.68 & 84 & 7.07 \\ & 220 & 17.84 \pm 1.08 & 0.65 & 234 & 11.19 \\ \text { BLAP } & 53 & 11.37 \pm 0.41 & 1.00 & 148 & 12.60 \\ & 48 & 11.37 \pm 0.41 & 1.00 & 37 & 23.86 \\ & 13 & 8.47 \pm 0.58 & 0.74 & 212 & 11.50 \\ & \mathbf{9 1} & 6.73 \pm 0.64 & 0.59 & \mathbf{1 6 5} & 5.91 \\ & \mathbf{2 3 3} & 0.00 \pm 0.00 & 0.00 & \mathbf{1 4 8} & 2.88 \\ \text { GLU } & 150 & 14.65 \pm 2.79 & 1.00 & 161 & 10.82 \\ & 407 & 10.61 \pm 1.99 & 0.72 & 393 & 14.42 \\ & \mathbf{9} & 6.69 \pm 0.94 & 0.46 & \mathbf{4 6 3} & 5.97 \\ & \mathbf{4 5 2} & 6.31 \pm 2.58 & 0.43 & \mathbf{5} & 6.90 \\ & \mathbf{6 2 6} & 4.70 \pm 1.63 & 0.32 & \mathbf{6 3 8} & 5.25 \\ & \mathbf{6 5 5} & 3.54 \pm 0.42 & 0.24 & \mathbf{5 0 9} & 4.30 \\ & \mathbf{3 2 8} & 2.46 \pm 0.40 & 0.17 & \mathbf{5 4 3} & 6.29\end{array}$

${ }^{a}$ The closest tyrosine residue, \#Tyr, to each tryptophan is indicated with its inter-residue distance $d_{\mathrm{Trp}-\mathrm{N}, \mathrm{Tyr}-\mathrm{O}}$. Inter-residue distances were determined with Chimera from crystal structure data of the corresponding PBD files from RCSB Protein Data Base. Pairs with inter-residue distance below $6.5 \AA$ are indicated in bold.

neighboring tyrosine $(<6.5 \AA$, indicated in bold). To further visualize this effect, data in Figure 4 a show a comparison across enzymes for the degradation of Trp-containing peptides relative to the proximity to tyrosine. Data show a strong relationship of increasing degradation rate constants of Trpcontaining peptides, $k_{\text {rel, Trp, }}$ with increasing distance to tyrosine, $d_{\text {Trp-N, Tyr-O }}$, up to a distance of $6.5 \AA$. Beyond this distance, no dependency was observed and overall fast degradation was measured. This critical distance for (PC)ET between tryptophan and tyrosine in the studied model enzymes is in agreement with previous observations for other proteins. $^{20}$ These observations may be explained by a distance dependency of the kinetically favored tryptophan radical reduction to the parent tryptophan relative to other competitive reactions. Analogously, all corresponding Tyrcontaining peptides of the (PC)ET pairs showed significant decay. However, also other Tyr-containing peptides, not in proximity to tryptophan residues, degraded, and it can be assumed that other decay pathways, e.g., reactions with ${ }^{1} \mathrm{O}_{2}$, were involved (see Figure S15.1a).

Triplet State Tryptophan Mediates Disulfide Reduction. Triplet excited tryptophan, ${ }^{3} \operatorname{Tr}{ }^{*}$, can reduce disulfide bridges (cystine) to a thiolate, $\mathrm{RS}^{-}$, and thiyl radical, $\mathrm{RS}^{\bullet}$, in linear peptides and in macromolecular structures (Figure 1D). ${ }^{19,35-39}$ The dissociation of a disulfide bond can change the conformational integrity of the protein and likely affects the enzymatic function. Evidence for Trp-mediated disulfide reduction was found for inter-residue distances between the

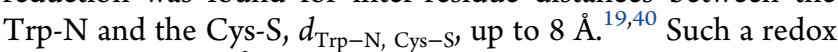
reaction between ${ }^{3} \mathrm{Trp} *$ and a disulfide would result in a faster decay of the Trp-containing peptide because the reaction competes with relaxation to ground state tryptophan. At the same time, the otherwise stable Cys-containing peptide will degrade. The aminopeptidase and phosphatase both feature cysteine residues forming disulfide bridges. Here, the disulfide bridge formed by Cys336 and Cys286 in phosphatase is the 

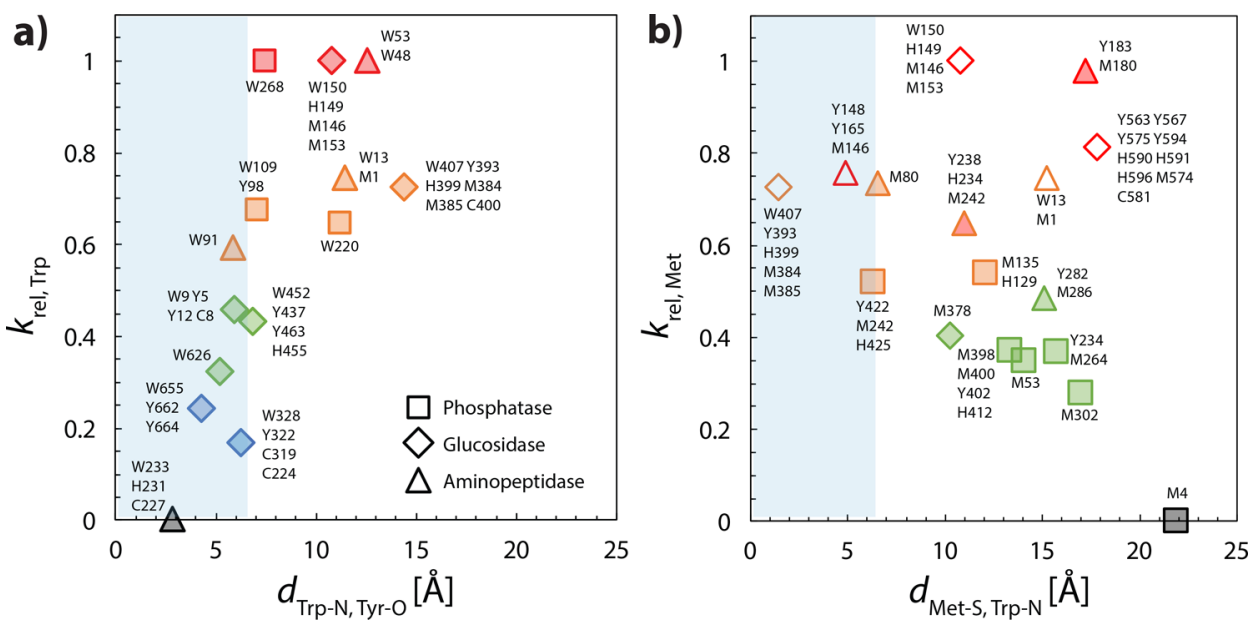

Figure 4. Relation between relative degradation rate constants, $k_{\text {rel }}$, and inter-residue distance, $d$ in $\AA$, for model enzyme phosphatase (ECAP, squares), aminopeptidase (BLAP, triangles), and $\alpha$-glucosidase (GLU, diamonds) illustrated for (a) the decay of Trp-containing peptides, $k_{\text {rel, }}$ Trp, against the inter-residue distance to the closest tyrosine residue, $d_{\mathrm{Trp}-\mathrm{N}, \text { Tyr-O }}$, and (b) the decay of Met-containing peptides, $k_{\mathrm{rel}, \text { Met }}$ against the inter-residue distance to the closest Trp-containing residue, $d_{\text {Met-S, Trp-N }}$. The open symbols indicate Met-containing peptides that are affected by degradation mechanisms other than methionine oxidation by triplet tryptophan. The colors of the symbols indicate increasing relative degradation rate constants from blue to red, and the number next to each symbol annotates the residues and their position in the primary amino acid sequence of the respective protein. The shaded backgrounds illustrate the critical distance for direct electron transfer reactions. Peptide degradation rate constants were normalized to the fastest decaying peptide in each enzyme to obtain $k_{\text {rel }}$.

only one in proximity to a $\operatorname{Trp}\left(d_{\operatorname{Trp}-\mathrm{N}, \mathrm{Cys}-\mathrm{S}}=8.16 \AA\right)$. In line with the expectations, these Cys-containing peptides degraded $\left(k_{\text {rel, Cys336 }}=0.44\right.$ and $\left.k_{\text {rel, Cys286 }}=0.27\right)$, and the tryptophan residue involved in the disulfide reduction, $\operatorname{Trp} 268$, is the fastest degrading peptide in phosphatase. The other two tryptophan residues not in proximity of a disulfide degrade significantly slower $\left(k_{\mathrm{rel}, \operatorname{Trp} 268}=1.00, k_{\mathrm{rel}, \operatorname{Trp} 209}=0.68[p=\right.$ $\left.0.012], k_{\text {rel, } \operatorname{Trp} 220}=0.65[p=0.0074]\right)$. The second disulfide bridge in phosphatase (Cys178 and Cys168) and Cyscontaining peptides in aminopeptidase do not show significant degradation $\left(k_{\mathrm{rel}}=0\right)$, and the closest tryptophan residues are more than $15 \AA$ away; hence, no ${ }^{3} \operatorname{Trp} *$-mediated reduction was expected (for details, see Table S15.1).

Triplet State Tryptophan Mediates Methionine Oxidation. Triplet excited tryptophan, ${ }^{3} \operatorname{Trp} *$, can also oxidize methionine, Met, by charge transfer (Figure $1 \mathrm{E}$ ). ${ }^{41}$ This pathway results in an unstable methionine radical cation intermediate, $\mathrm{Met}^{\bullet+}$, and a tryptophan radical anion, $\operatorname{Trp}^{\bullet-}$. When the residues are in close proximity to each other, both Trp- and Met-containing peptides experience faster decay. We investigated the dependence of degradation rate constants and proximity to tryptophan for all Met-containing peptides. Figure $4 \mathrm{~b}$ shows the relative reaction rate constant of all Met-containing peptides, $k_{\text {rel, Met }}$ and the respective distance to tryptophan, $d_{\text {Met-S, Trp-N }}$.

Several Met-containing peptides that diverge from the linear trend are affected by degradation mechanisms other than methionine oxidation (open symbols in Figure 4b). Four of these peptides contain a tryptophan or tyrosine residue, and their enhanced reactivities were discussed above. The fifth peptide is particularly long ( 42 residues) with eight photolabile amino acids, which may lead to enhanced degradation. Indeed, the data suggests a general trend of increasing degradation rate constants with decreasing inter-residue distances over a wide range of $20 \AA$ (closed symbols).

The apparent distance relationship over $20 \AA$ may point to a more complex network of subsequent reactions ultimately influencing the degradation of Met-containing peptides. It can be hypothesized that not only direct electron transfer reactions are taking place but also electron transfer assisted by neighboring residues and the protein backbone may be involved. ${ }^{20}$ Note that the distance measurements from the crystal structures alone may not be identical to those distances under aqueous conditions. While the absolute distances may differ, we see strong correlations of reactivity that are related to the spatial proximity of these residues.

Intramolecular Singlet Oxygen Formation. Our data suggest that the five photolabile amino acids (Trp, Tyr, Cys, Met, and His) are likely the dominant targets, not only of direct photochemical reactions but also for subsequent reactions initiated by tryptophan photochemistry. We observe, across the three enzymes, that peptides without photolabile residues consistently show much slower degradation $\left(k_{\text {rel }} \leq\right.$ 0.3 ). However, peptides containing photolabile amino acids exhibit a wide range of $k_{\text {rel }}$ values, which points out the heterogeneous degradation among these peptides (see Figure S15.2). In the previous sections, we demonstrated that photochemically excited proteinogenic ${ }^{3} \operatorname{Trp}^{*}$ can react directly with neighboring residues. In addition, ${ }^{3} \mathrm{Trp} *$ can form intramolecular ${ }^{1} \mathrm{O}_{2}$ by energy transfer to molecular oxygen ${ }^{42,43}$ and subsequently oxidize other photolabile amino acids. ${ }^{41,44-46}$ Although ${ }^{1} \mathrm{O}_{2}$ is only formed from ${ }^{3} \mathrm{Trp} *$ with a low quantum yield $\left(\Phi^{{ }_{1}} \mathrm{O}_{2}=0.065^{18}\right)$, it may mediate reactions that enhance the degradation of these photolabile residues.

While histidine is a photolabile amino acid that exclusively reacts with ${ }^{1} \mathrm{O}_{2}$, no distance relationship to tryptophan within the protein structures could be observed (see Figure S15.1b). The lack of such relationship may be explained by relatively low and heterogeneously distributed ${ }^{1} \mathrm{O}_{2}$ concentrations. However, when the enzymes are exposed to higher ${ }^{1} \mathrm{O}_{2}$ concentrations produced by an external source, we measured significant bimolecular inactivation rate constants with ${ }^{1} \mathrm{O}_{2}$ of $(3.10 \pm 0.01),(2.39 \pm 0.46)$, and $(11.21 \pm 0.05) \times 10^{7} \mathrm{M}^{-1}$ $\mathrm{s}^{-1}$ for phosphatase, aminopeptidase, and glucosidase, respectively. Further structural analysis is required to elucidate these site-specific reaction mechanisms. 


\section{IMPLICATIONS}

The optimized proteomics approach achieves high structural resolution of enzymes and allows one to identify site-specific molecular changes. By monitoring the individual protein fragments, one can generate site-specific fingerprints of degradation kinetics visualized in the three-dimensional protein model. The structural analysis can be used to test whether existing hypotheses generated using short linear peptides also take place in three-dimensional protein structures and how the microenvironment around residues affects reaction kinetics and pathways. The analysis of molecular damage of enzymes by an external stressor can be combined with functional analysis, e.g., analysis of enzymes activities. Here, the method was applied to study photoinactivation of extracellular enzymes. The holistic structural analysis revealed heterogeneous damage across the proteins. While the initial targets of direct photochemical reactions are the chromophoric tryptophan residues, subsequent redox reactions are closely related to the microenvironment around tryptophan and degradation rate constants correlate with inter-residue distances for all three model enzymes.

The improved resolution and detection of proteins has received increasing attention mostly to tackle biochemical and biomedical research questions. The presented work exemplifies an application for the field of environmental chemistry to study reaction pathways during inactivation of aquatic enzymes when exposed to light. The analytical approach can be applied to examine a variety of open questions in environmental sciences where the integrity of a protein is critical for its function. For example, damage from photochemically produced reactive intermediates, such as triplet excited organic matter and reactive oxygen species, can now be studied with respect to the inactivation of aquatic enzymes. Beyond enzyme inactivation, oxidative damage of virus capsid proteins during water treatment or irreversible interactions of organic pollutants with protein surfaces could be inspected by such structural analysis. Overall, reactions that involve modifications of amino acid side chains or formation of a covalent bond to the protein structure can be resolved with the presented proteomics approach.

\section{ASSOCIATED CONTENT}

\section{S Supporting Information}

The Supporting Information is available free of charge on the ACS Publications website at DOI: 10.1021/acs.est.7b06439.

Additional information and data on activity assays, proteomics workflows, data analysis, complementary color maps, and chromatograms and spectra for all detected peptides (PDF)

\section{AUTHOR INFORMATION}

\section{Corresponding Author}

*E-mail: elisabeth.janssen@eawag.ch; phone: +41 58765 5802.

\section{ORCID $\odot$}

Christine M. Egli: 0000-0002-0036-0562

Elisabeth M.-L. Janssen: 0000-0002-5475-6730

\section{Notes}

The authors declare no competing financial interest.

\section{ACKNOWLEDGMENTS}

We gratefully acknowledge support of the Swiss National Science Foundation (Grant number 200020-159809) and thank Kristopher McNeill for valuable discussions and Joanna Houska and Alexandra Büchler for their laboratory work.

\section{REFERENCES}

(1) Arnosti, C. Microbial extracellular enzymes and the marine carbon cycle. Annu. Rev. Mar. Sci. 2011, 3, 401-425.

(2) Sinsabaugh, R. L.; Follstad Shah, J. J.; Hill, B. H.; Elonen, C. M. Ecoenzymatic stoichiometry of stream sediments with comparison to terrestrial soils. Biogeochemistry 2012, 111, 455-467.

(3) Arnosti, C.; Bell, C.; Moorhead, D. L.; Sinsabaugh, R. L.; Steen, A. D.; Stromberger, M.; Wallenstein, M.; Weintraub, M. N. Extracellular enzymes in terrestrial, freshwater, and marine environments: perspectives on system variability and common research needs. Biogeochemistry 2014, 117, 5-21.

(4) Keith, S. C.; Arnosti, C. Extracellular enzyme activity in a riverbay-shelf transect: variations in polysaccharide hydrolysis rates with substrate and size class. Aquat. Microb. Ecol. 2001, 24, 243-253.

(5) Steen, A. D.; Arnosti, C. Long lifetimes of $\beta$-glucosidase, leucine aminopeptidase, and phosphatase in arctic seawater. Mar. Chem. 2011, 123, 127-132.

(6) Scully, N. M.; Tranvik, L. J.; Cooper, W. J. Photochemical effects on the interaction of enzymes and dissolved organic matter in natural waters. Limnol. Oceanogr. 2003, 48, 1818-1824.

(7) Espeland, E. M.; Wetzel, R. G. Complexation, stabilization, and UV photolysis of extracellular and surface-bound glucosidase and alkaline phosphatase:Implications for biofilm microbiota. Microb. Ecol. 2001, 42, 572-585.

(8) Boavida, M. J.; Wetzel, R. G. Inhibition of phosphatase activity by dissolved humic substances and hydrolytic reactivation by natural ultraviolet light. Freshwater Biol. 1998, 40, 285-293.

(9) Bent, D. V.; Hayon, E. Excited state chemistry of aromatic amino acids and related peptides. III. Tryptophan. J. Am. Chem. Soc. 1975, 97, 2612-2619.

(10) Pattison, D. I.; Rahmanto, A. S.; Davies, M. J. Photo-oxidation of proteins. Photochem. Photobiol. Sci. 2012, 11, 38-53.

(11) Janssen, E. M. L.; McNeill, K. Environmental photoinactivation of extracellular phosphatases and the effects of dissolved organic matter. Environ. Sci. Technol. 2015, 49, 889-896.

(12) Janssen, E. M. L.; Erickson, P. R.; McNeill, K. Dual roles of dissolved organic matter as sensitizer and quencher in the photooxidation of tryptophan. Environ. Sci. Technol. 2014, 48, 4916-4924.

(13) Grosvenor, A. J.; Morton, J. D.; Dyer, J. M. Profiling of residuelevel photo-oxidative damage in peptides. Amino Acids 2010, 39, 285296.

(14) Reece, S. Y.; Stubbe, J.; Nocera, D. G. pH dependence of charge transfer between tryptophan and tyrosine in dipeptides. Biochim. Biophys. Acta, Bioenerg. 2005, 1706, 232-238.

(15) Cai, S.; Fujii, N.; Saito, T.; Fujii, N. Simultaneous ultraviolet Binduced photo-oxidation of tryptophan/tyrosine and racemization of neighboring aspartyl residues in peptides. Free Radical Biol. Med. 2013, 65, 1037-1046.

(16) Stevenson, K. L.; Papadantonakis, G. A.; LeBreton, P. R. Nanosecond UV laser photoionization of aqueous tryptophan: temperature dependence of quantum yield, mechanism, and kinetics of hydrated electron decay. J. Photochem. Photobiol., A 2000, 133, 159-167.

(17) Vanhooren, A.; Devreese, B.; Vanhee, K.; Van Beeumen, J.; Hanssens, I. Photoexcitation of tryptophan groups induces reduction of two disulfide bonds in goat $\alpha$-lactalbumin. Biochemistry 2002, 41, 11035-11043.

(18) Tsentalovich, Y. P.; Snytnikova, O. A.; Sagdeev, R. Z. Properties of excited states of aqueous tryptophan. J. Photochem. Photobiol., A 2004, 162, 371-379.

(19) Vanhooren, A.; De Vriendt, K.; Devreese, B.; Chedad, A.; Sterling, A.; Van Dael, H.; Van Beeumen, J.; Hanssens, I. Selectivity of 
tryptophan residues in mediating photolysis of disulfide bridges in goat $\alpha$-lactalbumin. Biochemistry 2006, 45, 2085-2093.

(20) Chen, X.; Zhang, L.; Zhang, L.; Wang, J.; Liu, H.; Bu, Y. Proton-regulated electron transfers from tyrosine to tryptophan in proteins: Through-bond mechanism versus long-range hopping mechanism. J. Phys. Chem. B 2009, 113, 16681-16688.

(21) Lundeen, R. A.; McNeill, K. Reactivity differences of combined and free amino acids: Quantifying the relationship between threedimensional protein structure and singlet oxygen reaction rates. Environ. Sci. Technol. 2013, 47, 14215-14223.

(22) Navarro, E.; Robinson, C. T.; Wagner, B.; Behra, R. Influence of ultraviolet radiation on UVR-absorbing compounds in freshwater algal biofilms and Scenedesmus Vacuolatus cultures. J. Toxicol. Environ. Health, Part A 2007, 70, 760-767.

(23) Gil-Allué, C.; Schirmer, K.; Tlili, A.; Gessner, M. O.; Behra, R. Silver nanoparticle effects on stream periphyton during short-term exposures. Environ. Sci. Technol. 2015, 49, 1165-1172.

(24) Appiani, E.; Ossola, R.; Latch, D.; Erickson, P.; McNeill, K. Aqueous singlet oxygen reaction kinetics of furfuryl alcohol: Effect of temperature, $\mathrm{pH}$, and salt content. Environ. Sci.: Processes Impacts 2017, 19, 507-516.

(25) Geeraerd, A. H.; Valdramidis, V. P.; Van Impe, J. F. GInaFiT, a freeware tool to assess non-log-linear microbial survivor curves. Int. J. Food Microbiol. 2005, 102, 95-105.

(26) Ivanov, A. R.; Lazarev, A. V. Sample Preparation in Biological Mass Spectrometry; Springer: Dordrecht, Netherlands, 2011.

(27) Reddy, P. M.; Hsu, W.-Y.; Hu, J.-F.; Ho, Y.-P. Digestion completeness of microwave-assisted and conventional trypsincatalyzed reactions. J. Am. Soc. Mass Spectrom. 2010, 21, 421-424.

(28) MacLean, B.; Tomazela, D. M.; Shulman, N.; Chambers, M.; Finney, G. L.; Frewen, B.; Kern, R.; Tabb, D. L.; Liebler, D. C.; MacCoss, M. J. Skyline: an open source document editor for creating and analyzing targeted proteomics experiments. Bioinformatics 2010, 26, 966-968.

(29) Pettersen, E. F.; Goddard, T. D.; Huang, C. C.; Couch, G. S.; Greenblatt, D. M.; Meng, E. C.; Ferrin, T. E. UCSF Chimera-A visualization system for exploratory research and analysis. J. Comput. Chem. 2004, 25, 1605-1612.

(30) Stec, B.; Holtz, K. M.; Kantrowitz, E. R. A revised mechanism for the alkaline phosphatase reaction involving three metal ions. $J$. Mol. Biol. 2000, 299, 1303-1311.

(31) Desmarais, W.; Bienvenue, D. L.; Bzymek, K. P.; Petsko, G. A.; Ringe, D.; Holz, R. C. The high-resolution structures of the neutral and the low $\mathrm{pH}$ crystals of aminopeptidase from Aeromonas proteolytica. JBIC, J. Biol. Inorg. Chem. 2006, 11, 398-408.

(32) Golan, G.; Shallom, D.; Teplitsky, A.; Zaide, G.; Shulami, S.; Baasov, T.; Stojanoff, V.; Thompson, A.; Shoham, Y.; Shoham, G. Crystal structures of Geobacillus stearothermophilus $\alpha$-glucuronidase complexed with its substrate and products: Mechanistic implications. J. Biol. Chem. 2004, 279, 3014-3024.

(33) Land, E. J.; Truscott, T. G. Triplet excited state of coumarin and 4' 5 ' dihydropsoralen: Reaction with nucleic acid bases and amino acids. Photochem. Photobiol. 1979, 29, 861-866.

(34) Lu, C.-Y.; Liu, Y.-Y. Electron transfer oxidation of tryptophan and tyrosine by triplet states and oxidized radicals of flavin sensitizers: a laser flash photolysis study. Biochim. Biophys. Acta, Gen. Subj. 2002, 1571, 71-76.

(35) Dose, K. The photolysis of free cystine in the presence of aromatic amino acids. Photochem. Photobiol. 1968, 8, 331-335.

(36) Creed, D. The photophysics and photochemistry of the nearUV absorbing amino acids. - III. Cystine and its simple derivatives. Photochem. Photobiol. 1984, 39, 577-583.

(37) Davies, M. J.; Forni, L. G.; Shuter, S. L. Electron spin resonance and pulse radiolysis studies on the spin trapping of sulphur-centered radicals. Chem.-Biol. Interact. 1987, 61, 177-188.

(38) Hawkins, C. L.; Davies, M. J. Generation and propagation of radical reactions on proteins. Biochim. Biophys. Acta, Bioenerg. 2001, 1504, 196-219.
(39) Abaskharon, R. M.; Gai, F. Direct measurement of the tryptophan-mediated photocleavage kinetics of a protein disulfide bond. Phys. Chem. Chem. Phys. 2016, 18, 9602-9607.

(40) Permyakov, E. A.; Permyakov, S. E.; Deikus, G. Y.; MorozovaRoche, L. A.; Grishchenko, V. M.; Kalinichenko, L. P.; Uversky, V. N. Ultraviolet illumination-induced reduction of $\alpha$-lactalbumin disulfide bridges. Proteins: Struct., Funct., Genet. 2003, 51, 498-503.

(41) Hug, G. L.; Bobrowski, K.; Kozubek, H.; Marciniak, B. Photooxidation of methionine derivatives by the 4-carboxybenzophenone triplet state in aqueous solution. Intracomplex proton transfer involving the amino group. Photochem. Photobiol. 1998, 68, 785-796.

(42) Chin, K. K.; Trevithick-Sutton, C. C.; McCallum, J.; Jockusch, S.; Turro, N. J.; Scaiano, J. C.; Foote, C. S.; Garcia-Garibay, M. A. Quantitative determination of singlet oxygen generated by excited state aromatic amino acids, proteins, and immunoglobulins. J. Am. Chem. Soc. 2008, 130, 6912-6913.

(43) Davies, M. J. Singlet oxygen-mediated damage to proteins and its consequences. Biochem. Biophys. Res. Commun. 2003, 305, 761770

(44) Boreen, A. L.; Edhlund, B. L.; Cotner, J. B.; McNeill, K. Indirect photodegradation of dissolved free amino acids: The contribution of singlet oxygen and the differential reactivity of DOM from various sources. Environ. Sci. Technol. 2008, 42, 54925498.

(45) Davies, M. J.; Truscott, R. J. W. Photo-oxidation of proteins and its role in cataractogenesis. J. Photochem. Photobiol., B 2001, 63, $114-125$.

(46) Castaño, C.; Dántola, M. L.; Oliveros, E.; Thomas, A. H.; Lorente, C. Oxidation of tyrosine photoinduced by pterin in aqueous solution. Photochem. Photobiol. 2013, 89, 1448-1455. 